\title{
An Overview of Ebola Viral Disease
}

\author{
Article by Muekara Friday Dugbor \\ Medicine, Texila American University, Nigeria \\ E-mail: muekara@yahoo.com
}

\section{Epidemiology/Introduction}

Ebola virus (EBOV) and Marburg virus (MARV). Members of the Filoviridae virus family, are known as emerging and re-emerging zoonotic pathogens causing acute hemorrhagic fever with high case-fatality rate in human (up to $90 \%$ ) (1).

Ebola hemorrhagic fever (EHF) was first reported in 1976 during the Ebola outbreak in the Democratic Republic of the Congo (formally Zaire), and the virus is named after the Ebola River where it was discovered. Since then, 21 additional Ebola virus disease (EVD) outbreaks among human have occurred in the tropical regions of sub-Sharan Africa. The largest one to date took place in the Gulu District of Uganda in 2000-2001 caused by Sudan virus (SUDV). This outbreak resulted in 425 cases, of which 216 were laboratory confirmed, and the overall case fatality rate was 53\% (20. The Ebola strain that is now circulating in West Africa bears shows the homology of 97\% with Zaire Ebola virus samples found in the Democratic Republic of Congo and Gabon(2). Historically, this strin has caused the highest mortality (90\%), while the current estimate of case fatality rate is less than $60 \%$ (3).

During December 2013, the epidemic of EVD started in Guinea(2), and the World Health Organization (WHO) received official notification of a rapidly evolving outbreak of EVD on March 23, 2014, in August 2014, WHO declared this to be a "public Health Emergency of international concern"(3). In midSeptember 2014 , the case fatality rate among patients with definitive outcomes was $70.8 \%$ (95\% confidence interval (CI), 68.6 to 72.8) and was consistent among Guinea, Liberia, and Sierra Leone. Nigeria's case fatality rate was lower at $45.5 \%$, although the current estimate is based on only 11 recent cases. The in-hospital case fatality rate was $64.3 \%$ (95\% CI, 61.5 to 67.0), which was lower than those for all patients with definitive outcomes, and this rate was consistent among countries. A range of $56.1 \%$ (95\% CI, 41.0 to 70.1) in Guinea to $80.0 \%$ (95\% CI, 68.7 to 87.9) in Liberia of health care workers died. Despite multinational and multisectoral responses to the disease, a growing number of new cases and death were reported every week (4).

There is no change in the control in the measures for this epidemic and by November 2, 2014, the cumulative reported numbers of Ebola confirmed and suspected cases for Guinea, Liberia, and Sierra leone are predicted to be 5740, 9890, and 5000, respectively, exceeding 20000 cases in total(4). The majority of cases are between 15 to 44 years old (49.9\% male). In terms of reported morbidity and mortality, the current EVD epidemic is much greater than all previous outbreaks combined. The real number of those who have been infected and died is likely much higher (4).

This time, the outbreak has become so large that the three most affected countries, namely, Guinea, Liberia, and Sierra Leone, face numerous challenges for the implementation of rigorous control measures at the scale needed to prevent transmission and to supply all EVD patients with clinical care(4).

\section{Species of ebola virus}

The genus Ebola virus is classified into five different virus: SUDV, Tai Forest virus, Reston virus, EBOV, and Bundibugyo virus. Among them, EBOV causing the EHF is associated with the highest fatality rate in humans (57\%-90\%), followed by SUDV (41\%-65\%) and Bundibugyo virus (40\%). To date, Tai Forest virus has only been known to cause two nonfatal human infections, while Reston virus cause asymptomatic infection in humans. 
The viral hemorrhagic fever (VHFS) represent a group of diverse animal and human diseases caused by RNA virus belonging to four distinct families including Arenaviridae, Filoviridae, Bunyaviridae and Flaviviridae. The severity and clinical symptoms of VHFs may significantly change depending on different factors: the type of causative agent, and the epidemiological and clinical features of host.

In general, all patients shows evidences of fever and coagulation abnormalities that may lead to disseminated intravascular coagulation, multiple organ failure, signs of shock and eventually death. The VHF can be severe and life-threatening, and it may occur as isolated cases, such as cases imported from endemic areas, or may cause a devastating lethal outbreak. Human sporadic and outbreak cases have been reported with high case-fatality rate, involving social and economic disruption

\section{Structures}

Filo viruses are enveloped particles with a non-segmented, single stranded, negative-sense RNA genome, approximately $19 \mathrm{~kb}$ in size. EBOV and MARV genomes encode seven structural proteins, and also EBOV encodes two nonstructural soluble glycoproteins (GP): soluble GP and small soluble GP. All known MARV strains consists of one Species Lake Victoria Marburg virus, while EBOV strains consist of four different species: Zaire Ebola virus

(ZEBOV), Sudan Ebola virus (SEBOV), Cote d'Ivoire Ebola virus (CIEBOV) and Reason Ebola virus (REBOV). The newly discovered Bundibugyo Ebola virus (EEBOV) has been proposed as the fifth species. The species vary in their apparent pathogenicity in human; ZEBOV is the most pathogenic (up to 90\% case fatality rate), followed by SEBOV (approximately 50\% case fatality rate) and BEBOV cause lethal infections in nonhuman primates, but not being associated with fatal human cases yet(1,8). By systematic viral replication, EBOV and MARV result in the release of high levels of inflammatory cytokines, coagulation abnormalities and fluid distribution problems. These process are observed as hemorrhage and leakage; ultimately these may lead to multiple organ failure and shock $(9,10)$.

ZEBOV was first discovered in 1976, being the most virulent species with case fatality rates in humans up to $90 \%$ and as high as $100 \%$ lethality in experimental macaque models, the current gold standard animal model for ZEBOV among other established models (11).

\section{Reservoir}

Recent evidence has confirmed the importance of bats as potential reservoir species of filo viruses; however, it is unclear whether other species are also involved or how transmission to human and/or apes takes place. EHF is believed to persist in a reservoir species generally found in endemic places. Apes, man, and perhaps other mammalian species being susceptible to Ebola virus infection are considered as end hosts of Ebola rather than as reservoir.

Although wide efforts have been made to find the natural reservoirs in large outbreaks of EHF, neither potential hosts nor arthropod vectors have been identified. For a long time, rodents and bats have been regarded as potential reservoir species. This was proved by experimental studies in African plants and animals that confirmed the transmission of productive infection of African fruit and insectivorous bats with ZEBOV, though a firm link could not be achieved. The inspection for potentially vectors, especially among arthropods has been always negative, including bedbugs (cimex hemipterus) captured in the beds of infected persons.

\section{Transmission}

Presumably, most index cases become infected through contact with an infected animal. While planning defenses against bio warfare agents such as filo viruses, it is important to consider that respiratory portal of entry is the most likely route of dissemination of agents such as aerosols.

The virus is transmitted to people as a result of direct contact with body fluids containing virus (vomitus, sweat, stool, urine, tears, breast milk, saliva and respiratory secretions) of an infected patient during the acute stage of disease). 
Epidemiological studies have revealed that family members are at risk of infection because they may come in contact with infected body fluids or may help to prepare the corpse of an infected person for burial. Direct contact with virus containing material from contaminated hands of caregivers to their own mouth or eyes is the most common cause. Caregivers who work both at home and in the hospitals are at greatest risk for exposure.

While studies have proved the spread of EBOV and MARV via aerosol particles under controlled laboratory conditions such transmission rarely appeared in humans in a hospital or household setting during epidemics.

Further, infection can occur through sexual contact and the virus has been traced in semen for up to seven weeks after recovery. It is recommended to control and use condoms during intercourse, and to avoid breast feeding for at least three months after recovery to prevent secondary cases. The center for Disease Control and Prevention (CDC) has clearly outlined isolation procedures (3).

The spread of infections are also the product of nosocomial or occupational transmission. For instance, in the first epidemics of Ebola, Zaire, in 1976, the usage of contaminated needles resulted in simultaneous out break among over hundreds patients. Another example covers spread of the virus to an entire surgical team who performed an exploratory laparotomy on an infected patient in Kikwit in 1995

In fact health care workers coming in contact with affected people were mostly consisted as the first generation causes in previous outbreaks. The propagation of infectious diseases can be avoided among health care workers through early detections of subjects and enforcement of appropriate preventive practices. Historically, outbreaks have gradually burned themselves out or have been controlled by effective public health measures including isolation of sick individuals and appropriate barrier protection methods for care providers and funeral services. It is believed that transmission of viruses needs direct contact or contact with infectious fluids rather than a possible aerosol route of transmission.

EBOV and MARV are regarded as re-emerging and highly infectious pathogens. Outbreaks have been associated with human sporadic cases, involve high rates of cases-fatality and cause social and economic disruption. The substantial appearance of both EBOV and MARV with severe hemorrhaging in most cases has also contributed to the high transmission rate and the fear of epidemic and imported cases. According to the US CDC, EBOV and MARV have been classified as category a bioterrorism agents due to their highly infectious nature and potential use in biological weapon.

\section{Clinical aspects}

Before the recent epidemic in West Africa, past EVD outbreaks in central Africa had been limited in size and geographic spread, typically affecting one to a few hundred persons, often residing in remote forested areas.

Recognizing the signs of EVD is challenging, the incubation period usually last 5 to 7 day although it can be as short as 2 days and as long as 21 days. Approximately $95 \%$ of the patients appear signs within 21 days after exposure which is the recommended period for follow-up contacts. In general, blood samples start to be tested positive by polymerase chain reaction-based diagnostics one day before symptoms onset.

Typical features include fever, profound weakness, diarrhea, abdominal pain, cramping, nausea and vomiting for 3-5 days and may be persisting for up to a week. Some patients may also have pharyngitis and maculopapular rash. Laboratory complications including elevated aminotransferase levels, marked lymphocytopenia, and thrombocytopenia may have occurred.

Clinical EHF is featured by sudden onset of fever, fatigue, chills, general malaise, headaches, myalgia, anorexia and gastrointestinal distress within 3-13 days following exposure to virus. Many patients develop hemorrhagic manifestations from which the term "hemorrhagic fever" has been derived.

Hemorrhagic fever occurs in less than half of infested subjects and gross bleeding is relatively rarer. The most common signs reported between symptom appearance and case detection include fever (87.1\%), fatigue (76.4\%), loss of appetite (64.5\%), vomiting (67.6\%), diarrhea (65.6\%), headache (53.4\%) and 
abdominal pain (44.3\%). Certain hemorrhagic features were rarely reported (in $<1 \%$ to $5.7 \%$ of patients); however, unexplained bleeding was reported in $18.0 \%$ of cases.

These patterns are the same for all countries. Bleeding takes place most commonly in the gastrointestinal tract and may demonstrate as melena, petechial, conjunctival hemorrhage, hematuria, easy bruising, or intraperitoneal bleeding. Mucous membrane bleeding, failure venipuncture sites and excessive clot formation have also been described.

These symptoms progress over the time and patients suffer from dehydration, stupor, confusion, hypotension and multi-organ failure, leading to fulminant shock and eventually death. Fatal causes tend to develop early clinical signs during the infection and death often occurs between the sixth and sixteenth days of illness.

\section{Pathogenesis and laboratory abnormalities}

At the entry site into the body, MARV and EBOV are capable to infect macrophages and other cells of the phagocytic system. Macrophages in vitro are highly susceptible to infect and produce a large number of viral particles, and hence serve as a vehicle to deliver the virus to a variety of organ systems such as liver, endothelium, spleen, lymph nodes, kidney, adrenal gland, and pancreas.

Marked leucopenia with a left shift and atypical lymphocytes can be observed on peripheral smears of infected patients.

Since lymphocytes are not assumed to be host targets for the virus, a substantial reduction in the number of lymphocytes is supposed as a result of bystander apoptosis, showing the death of a large number of lymphocytes triggered by mediators which are released from virus infected target cells and/or secretion of viral GP. Impaired production of pro-inflammatory cytokines and impaired stimulation of $\mathrm{T}$ cells also play a role in this phenomenon.

EBOV seems to utilize multiple cellular pathways for entry into host cells. Potential key mechanism in neutralization cover direct inhabitation of GP attachment to cell surface or endosomal receptors and blocking fusion of the viral and host membranes. Preventing cathepsin-induced cleavage is another formal possibility, albeit controversial.

Through experimental infection of nonhuman primates (the gold standard animal model for Ebola virus infection), laboratory studies have known many aspects of the disease, because the signs and disease induced in these animals are similar to those appeared in humans.

Filo viruses lead to highly cytopathic effect and are capable of rapidly replicating to high viral doses in a range of cells and cause their lyses. Filo viruses enter via small skin lesions and mucus membranes from which a direct access to the vascular system is available. There, monocytes, macrophages and dendritic cells are infected in the early stage of the disease; and due to rapid spread of the virus through the organs, particularly in the spleen, liver, and lymph nodes, the spectrum of target cells increases to include endothelial cells fibroblasts, hepatocytes and many other cells. Consequently, critically ill patients display intensive viremia and antigenemia.

Extensive information on EHF pathophysiology have been obtained from the samples collected during the Gulu outbreak including the observation of aspartate aminotransferase, D-dimer, blood urea nitrogen and higher creatinine levels than normal, although calcium and albumin levels are less than normal in samples from fatal EHF cases. Fatal rates were also affected by elevated levels of the cytokines interleukin 6, interleukin 10 and macrophage inflammatory protein, further human leukocyte antigen B67 (HLA-B67), HLA-B15 and marked CD8 lymphopenia contributed to fatal outcomes, while HLA-B7and HLA-B14were associated with neonatal outcomes in humans.

\section{Molecular assays}

Ebola laboratory diagnosis can be achieved in two different ways: measuring the host-specific immune responses to infection and detection of viral particles, or particle components in infected individuals. 
Nowadays, RT-PCR and antigen detection ELISA are the main diagnostic assays for acute infections. Viral antigen and nucleic acid can be traced in blood from Day 3 up to Day 7-16 following systems begin.

The most general assays used for antibodies detection are direct IgG and IgM ELISAs and IgM capture ELISA. IbM antibodies can appear as early as two days following the onset of signs and disappear between 30 and 168 day after infection. IgG-specific antibodies develop between 6 and 18 days after illness onset and persist for a long time. An IgM or rising IgG titer (four-fold) contributes to strong presumptive diagnosis.

Considering the physiological kinetics of humoral response and since VHFs resulted in impaired antigen-presenting cell functions, antibody titers are low at least in the earlier stages of illness. Therefore, serology is often not the major diagnostic option in critical phase, but it can be a particular useful practice to confirm the diseases etiology in convalescent patients.

\section{Diagnosis}

For diagnosis, the best option is to have a comprehensive, relatively unspecific definition in accordance with both clinical (fever and other symptoms) and epidemiological (contact with a case) criteria. Due to poor specificity of the symptoms, it is difficult to practice clinical diagnosis at the beginning of the epidemics.

After identification of virus responsible for the outbreak, all suspected cases should be considered at high risk of exposure and the case definition and exposure risks must be included for better management of the epidemic.

Case definition of EVD includes index case: very first case (probable or confirmed) reported as the origin of the epidemic; alert case: person with sudden appearance of high fever or sudden death or bleeding diarrhea, or bleeding in urine; suspect case: person, dead or alive, who has (or had): (a) fever (>38.50C or $101.50 \mathrm{~F}$ ) with additional signs (severe headache, muscle pain, vomiting, diarrhea, abdominal pain, or unexplained hemorrhage) and (b) epidemiologic risk factors within the past 21 days before the start of symptoms (close contact with body fluids of a suspect or probable case of EVD, or direct handling of bush animals from disease-endemic areas; probable case: any person evaluated by a clinician, having symptoms compatible with EVD, or a dead person with an epidemiological link to a confirmed case contact person without suggestive symptoms of disease, but had unprotected contact with suspect or probable cases of EVD (living in the same house, providing care during the illness and participated in the burial rites). To assess the risk level is very important; confirmed case: cases who had positive laboratory response on the detection of either Ebola virus antigen or Ebola IgG antibody; not a case: person with specific detectable antibody or antigen for Ebola.

\section{Treatment and prevention}

A wide range of studies in vitro and several animal models have been developed for EBOV and MARV; however, currently neither a licensed vaccine nor an approved treatment is available. Scientists working in high containment facilities, health care workers in Africa and people residing in the affected areas in Africa run a risk of potential exposures. In the occurrence of bioterrorism acts involving filo viruses, the high-risk population could be quite extensive. Thus, as an important part of contingency plans, counter measures are developed.

Passive transfer of serum collected from survivors of Junín virus or Lassa virus has confirmed affected provided therapies which are begum quickly following the infection. However, the experiment of antibody passive transfer has highly failed to treat filo virus infectious. During a 1995 outbreak of EBOV in Kikwit, Democratic Republic of the Congo, seven of eight patients with symptoms and detectable EBOV antigen in their blood who received whole blood from convalescent EBOV patients survived.

The lethality rate $(12.5 \%)$ from this treatment was significantly lower than the overall case fatality rate (80\%) for the EBOV epidemic; however, it is often difficult to interpret the role of antibodies in the 
achieved protection since the patients received whole blood, not just antibodies, from recovering patients in the additional hospital care. After the 1995 epidemic,

WHO product a commercially available equine IgG product from horses hyper vaccinated with EBOV for potential use in human. Similar IgG preparations had been used with reported success for hamadryas baboons, in which this antibody protected against lethal EBOV challenge. In contrast, the commercially available equine IgG did not fully protect cynomolgus macaques against $\mathrm{EBOV}$, although clinical feature onset, viremia levels and time to death were delayed relative to the controls.

Recently, a great attention has been paid unlicensed treatments and vaccines. A "cocktail" of humanized-mouse antibodies (ZMapp) is among the therapies in development, showing promise in nonhuman primates. Two US citizens who recently evacuated from Liberia to Atlanta were given ZMapp and both patients demonstrated clinical improvements. Other candidate therapeutics covers RANApolymerase inhibitors and small interfering RNA Nano particles that are inhibitors of production.

The results obtained from gene-silencing treatment using small interfering RNAs have been good both in guinea pigs and non-human primate models of Ebola infections. This data suggests that RNA interference may be an effective post-exposure treatment strategy for people infected with Ebola virus and perhaps other VHF agents. Unfortunately, production and cost issues can substantially constraint the current use of this approach.

Preclinical evaluated is also underway for various vaccine candidates. One is a chimpanzee adenovirus vector vaccine, into which two Ebola gene encoding glycoproteins have been inserted. Two other vaccine candidates involve vesicular stomatitis virus pseudo types. Human clinical traits for one of these vaccine is planned to start in early 2015 .

In the past decade, many efforts have been made in the development of different vaccine platform and treatment strategies against filo virus. Though there is a lack of highly efficacious treatment options, multiple vaccine platforms have been developed with good efficacy against EBOV and MARV including virus-like particles, Venezuelan equine encephalitis virus replicons, replication incompetent adenovirus serotype 5 vectors, replication competent recombinant human Para influenza virus 3 and recombinant vesicular stomatitis virus (rVSV), all these platform have been assessed in the nonhuman primate models and proved to be protective.

Currently, the rVSV platform is one of the more promising vaccine approaches against filo virus. As a none segment, negative stranded RNA virus in the family Rhadovirus, VSV is primarily an animal pathogen, and no evidence is available for its role for acute illness in humans. Two serotypes, designated as serotypes New Jersey and Indiana, are known to be circulating on the American continent. Both are transmitted via mosquitoes, sand flies or black flies and cause a deadly effect. Two rVSV vaccine vectors have been extensively investigated over filo virus animal disease models: rVSV/ZEBOV. GP expressing the GP derived from ZEBOV strain Mayinga and rVSV/MARV-GP expressing the GP derived from MARV strain Muosokt.

The efficacy of rVSV/ZEBOV-GP in vaccine or post-exposure treatments has been tested through mouse-adapted ZEBOV in mice and hamsters, guinea pig-adapted ZEBV, SEBOV, CIEBOV and BEBOV in nonhuman primates. The researcher showed protective efficacy data of rVSV/MARV-GP against MARV infection. The protection for post-exposure rVSV treatment is still an unknown mechanism. Acting as a vaccine vector to induce very strong immune responses, VSV can overcome filo virus-driven suppression of this response, thus inhibiting filo virus replication and infection spread. It has been revealed that rVSV infect the same target cells as filo virus and the viral interference leads to a block in EBOV and MARV replication. Again, the development of a humeral non neutralizing immune response contributed in survival, but this is unlikely to be protective mechanism due to its late development.

At least, six different vaccine systems are promising complete protection for nonhuman primates against MARV or EBOV infection among those prospective vaccines with efficacy in nonhuman primate models of filo virus hemorrhagic fever; two options, one based on a replication-defective adenovirus 
serotype 5 and the other on rVSV, have shown complete protection to nonhuman primates when administered as a single injection.

There are no approved vaccines or antiviral therapeutics for MARV or EBOV currently available for human use. Although MARV or EBOV hemorrhagic fevers are rare diseases, vaccination could be an important preventive tool for several groups including risk groups during filo virus epidemics in affected regions in sub-Sahara Africa (medical personnel, patients care personnel, family members); national and international healthcare workers and outbreak response personnel; laboratory workers conducting research on filo viruses; military and other services personnel susceptible to filo virus used as bio weapons.

VSV is the prototypic member of the family Rhabdoviridae and a number of its certain characteristics are important for a vaccine vector, namely, replication in almost all known mammalian cell lines, growth to very high titers and a strong induction of innate and adaptive (humoral as well as cellular) immune responses.

Providing vaccine to people before traveling to endemic regions of the world could help prevent lifethreatening diseases. An effective preventive vaccine has the potential to defend against regional epidemics and reduce the likelihood of global transmission of filo virus infections. Studies on rhesus macaques prove that treatment with recombinant inhibitor of factor VIIa/tissue factor and activated protein $\mathrm{C}$ contributed to significant increased survival after the experimental infection with ZEBOV.

Activated protein $\mathrm{C}$, recombinant inhibitor of factor VIIa/tissue factor and modipafant might be considered in feature clinical experimental plans for severe dengue and/or Ebola infections in patients which are known to proceed through shock and not responsive to standard support treatments. Finally, it was found that transgenic mice expressing very high levels of human mannose binding lectin concentrations (a C-type lectin that recognizes hoxose sugar and acts as a first-line host defense against a wide range of viral pathogens) are more resistant to fatal Ebola infections than wild-type mice. This suggests that modulation of mannose binding lectin activity may be an interesting field for further clinical studies.

Ebola patients receive supportive care; no licensed therapy is known to be effective against the virus. Basic clinical supports consist of aggressive volume and electrolyte management, oral and intravenous nutritional therapy and medical interventions to control fever and gastrointestinal distress as well as to treat pains, anxiety and agitation. Diagnosis and treatment of concomitant infections and super infections including malaria and typhoid are also regarded as important aspects of patient care.

In the recent past, experimental post-exposure interventions against filo virus infections have consisted of hyper immune equine IgG, EBOV-specific human monoclonal IgG antibody, whole blood transfusions from convalescent survivors, recombinant interferon, recombinant nematode anticoagulant protein $\mathrm{C} 2$, recombinant human activated protein $\mathrm{C}, \mathrm{rVSV}$ vectors, small interfering RNANs and phosphorodiamidate morpholino oligomers.

These interventions can reduce the likelihood of early infections in humans; improve biological safety; provide infection-control training and equipment for hospitals and ambulance; decrease the number of epidemics; provide leadership for behavioral change involving safe burial practices and equipments; communicate with community members and health workers; reduce the spillover of zoonotic diseases into human populations; prevent contact between humans and bats; improve food security; and minimize dangerous handling of consuming bush meat. Three core treatments have contained all previous Ebola viruses and can stop this one as well: exhaustive case and contact finding, effective response to patients and the community and preventive interventions.

Laboratory experiment with RT-PCR provides sensitivity and specific and can return the results within some hours; the test is now becoming more popular and widely available in the affected areas. Responding to cases includes the treatment of patients while isolated, through contact tracing and monitoring all contact up to 21 days after exposure. It is difficult to isolate and treat people with EVD, not 
because the illness is particularly infectious or it is particularly hardy virus, but a single lapse can have devastating consequences.

Neither negative air flow nor special respirators are needed; it requires meticulous and scrupulous attention to guidance on gown, gloves, facemask and eye protection and great caution while removing protective equipments. Improvement in hospital infection control measures through the region would have a significant impact on the number of EVD and other diseases. Soap and water or alcohol-based hand sanitizers can disrupt the envelop of this single-stranded RNA virus, and dilute bleach effectively protects against contamination and are readily available even in remote settings.

Provision of supportive therapy especially fluid and electrolyte maintenance and treatment of bacterial super-infections can substantially improve survival rate, initiating identification of contacts and measure of people's temperature daily for 21 days following exposure are needed. There are three main preventions, and the first is to practice strict infection control measures in health care settings; the highest risk of transmission occurs among patients with delayed detection and isolation, not those with diagnosed infection. The second is to provide education and support for the community regarding modification of long-standing burial traditions aimed for preventing direct contact with the blood and body fluids of infectious people, at least temporarily, until the outbreak is controlled; and it will stop the second key medium of the virus widespread. This issue is culturally sensitive that requires culturally relevant and appropriate outreach and educational materials. The third is to avoid direct contacts with bush meat (wild animals hunted for sustenance) and bats (that may be primary natural host of Ebola virus) can eliminate the risk of early importation of Ebola virus into humans.

Health-care workers' knowledge and practices regarding the safe infection-control measures including an appropriate use of personal protective equipment offer protection to both workers and patients, because health care associated infection has been a major cause of transmission during previous outbreaks.

Suspected patients should be isolated immediately from other patients and barrier practices should be instituted. In addition, strict precaution should be taken when dealing with specimens to avoid propagation of the infection among caregivers. Precaution tools need to be consistently used, like gloves, gown, face shields, mask and eyewear. Further, the existing CDC guidelines recommend respiratory protection by using N-95 respirator. Cleaning and decontaminating surfaces and objects contacting with patients must be considered in order to prevent the transmission to health workers and family members.

\section{Conclusion}

EVD is a painful reminder that an outbreak anywhere can be a risk everywhere. The Global Health Security Agenda seeks to enforce public health systems in most affected countries in order to eliminate the spreads before they become emergencies. Although great improvement have been achieved over the past decade, better surveillance, real-time sharing of data and taking rapid action based on the available information remain necessary. Because Ebola virus is primarily transmitted through contact with the body fluids of symptomatic patients, the infection spread can be stopped by an early diagnosis, contact tracing, patient's isolation and care, infection control and safe burial.

\section{References}

[1]. Asian Pacific journal of Tropical Biomedicine.

[2]. Asian Pac J Trop Biomed2014; 4(9):673-4.

[3]. Ansari AA. Clinical features and pathobiology of Ebola virus infection. J Autoimmune 2014; 55:1-9.

[4]. Feldmann H, Geisbert TW. Ebola hemorrhagic fever. Lancert 2011; 33(9768):849-62

[5]. Hensley LE, Stevens EL, Yan SB, Geisbert JB, Macias WL, Larsen T, et al. Recombinant human activated protein $\mathrm{C}$ for the post exposure treatment of Ebola hemorrhagic fever. J Infect Dis 2007; 196(Suppl 2); S390-9.

[6]. Koenig KL, Majestic C, Burn MJ. Ebola virus disease: essential public health principles for clinicians. West J Emerg Med2014; 15(7):728-31. Fauci AS. Ebola-underscoring the global disparities in health care resources. NEngl J Med 2014; 371(12):1084-6. 
[7]. WHO manual on the treatment hemorrhagic fev Marzi A, Feldman H, Geisbert TW, Falzarano D. Vesicular stomatitis virus based vaccines for prophylaxis and treatment of filo viruses infections' Bioterror Biodef2011;doi:10.4172/2157-2526.SI-004.

[8]. Waheed Y. Ebolain West Africa: an internal medical emergency.

[9]. Wiwanitkit V. Ebola virus infection: what should be known? N Am J

[10]. Med Sci 2014; 6(11):549-52.

[11]. Stein RA. What is Ebola? Int J Clin Pract 2015;69(1):49-58

[12]. WHO Ebola response Team. Ebola virus disease in West Africa-the first 9 months of the epidemic and forward projections. N Engl J Med 2014; 371:1481-95. 